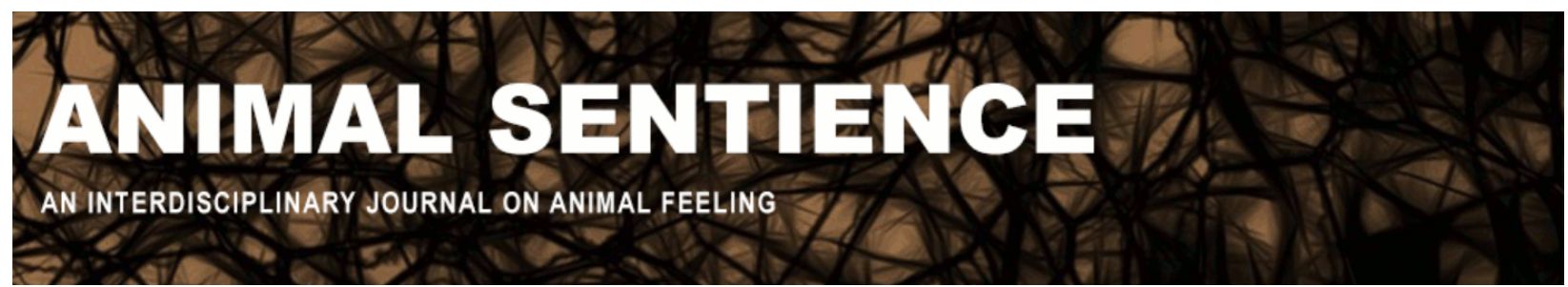

Merskin, Debra (2017) Getting to the other side. Animal Sentience 17(5) DOI: 10.51291/2377-7478.1224

Date of submission: 2017-10-13

Date of acceptance: 2017-10-17

(c) (i)




\title{
Getting to the other side
}

Commentary on Marino on Thinking chickens

\author{
Debra Merskin \\ School of Journalism \& Communication \\ University of Oregon
}

\begin{abstract}
Marino's comprehensive, detailed, and timely review provides clear evidence of the sentience of chickens and strong support for those wishing to challenge their exclusion from even the limited protections currently accorded to animals grown for food.
\end{abstract}

\begin{abstract}
Debra Merskin is Professor of Media Studies. Her work focuses on the intersection of race, gender, and species, particularly how nonhuman animals' species determines whether humans accord them compassion. Her forthcoming book, Re-presenting Species, examines how media portrayals influence human understanding of animals and animal well-being. Animalsandmedia.org
\end{abstract}

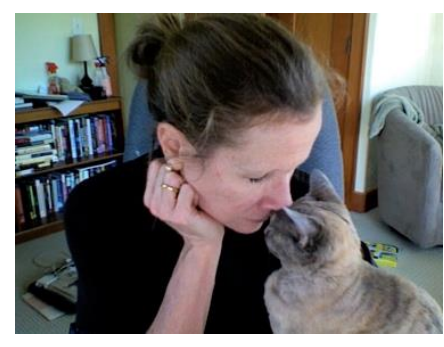

"To think" is defined by Merriam-Webster as "to form or have in the mind," "to have as an intention." Do animals other than humans think? Without a doubt. Do birds, and more specifically, chickens (Gallus gallus domesticus) think? This is one of the questions Marino $(2017 a, b)$ examines in this comprehensive review of the published academic literature about the cognitive, emotional, and behavioral abilities of these fascinating birds. She analyzes the findings in 16 major peer-reviewed journals, as well as book chapters, theses, and dissertations that use "terms relative to intelligence, cognition, and behavior" (p. 129). Search engines such as Web of Science Core Collection facilitated the data gathering. The two primary goals of the review are (1) to better understand chickens, and (2) to identify important areas for future, non-invasive, research.

After a discussion of chicken evolution, phylogeny, and domestication, the cumulative findings are presented under headings such as "visual and spatial orientation," "numerical abilities," "time perception," "self-control," "emotions," and others. Marino reviews publications according to 14 categories (with subcategories) for findings about the "cognitive, emotional, personality, and social characteristics of domestic chickens" (p. 129). The analysis reveals that these birds - long regarded, particularly by those who wish to grow and consume them, as "dumb" - engage in many of the same socially complex behaviors and communicate in ways similar to other birds who are traditionally deemed more intelligent, as well as many mammals. Chickens process what they see, even if objects are partly and sometimes completely occluded; they have numerical abilities, sense and anticipate time, remember, exert self-control, reason and infer logically, are self-aware, and communicate referentially. Via evoked representations, 
chickens are socially and individually complex, empathetic, and socially discriminatory. In addition, chickens are emotional, engage in social learning processes, and have distinct personalities. Taken together the findings reveal what those interested in the continued breeding, confinement, transport, and killing of these birds for food and other purposes would rather educators, legislators, consumers, and citizens did not know - that chickens are thinking, feeling, and communicating individuals.

This study has significant implications for policy makers, animal activists, and consumer citizens. Every year at least 9 billion chickens are killed for food in the United States and 305 million are used to produce eggs (peta.org). Their natural life expectancy is 6 to 12 years. Those killed for food, their growth accelerated by selective breeding and genetic manipulation, live approximately 6 to 7 weeks. Laying hens are confined to small cages for up to three years or until they stop producing and then they are killed. The Humane Slaughter Act (1958, rev. 1978), "requires the proper treatment and humane handling of all food animals slaughtered in USDA inspected slaughter plants" (USDA). This Act does not apply to chickens (or other birds). Thus, how they are grown, transported, confined, and killed is not regulated. Marino's comprehensive,

detailed, and timely review thus provides clear evidence of the sentience of chickens and strong support for those wishing to challenge their exclusion from even the limited protections currently accorded to animals grown for food.

\section{References}

Chickens used for food. Peta. https://www.peta.org/issues/animals-used-for-food/factoryfarming/chickens/

Humane Methods of Slaughter Act (1958/1978). United States Department of Agriculture.

Marino, L. (2017a). The inconvenient truth about thinking chickens. Animal Sentience 17(1).

Marino, L. (2017b). Thinking chickens: A review of cognition, emotion, and behavior in the domestic chicken. Animal Cognition, 20: 127-147. 